\title{
Homeopathic and conventional treatment for acute respiratory and ear complaints: A comparative study on outcome in the primary care setting
}

\author{
Max Haidvogl ${ }^{\dagger 1}$, David S Riley ${ }^{\dagger 2}$, Marianne Heger ${ }^{\dagger 9,3}$, Sara Brien ${ }^{\dagger 4}$, \\ Miek Jong*5, Michael Fischer ${ }^{\dagger 6}$, George T Lewith ${ }^{\dagger 4}$, Gerard Jansen ${ }^{\dagger 7}$ and \\ André E Thurneysen ${ }^{\dagger 8}$
}

\begin{abstract}
Address: ${ }^{1}$ Ludwig Boltzmann Institute for Homeopathy, Graz, Austria, ${ }^{2}$ University of New Mexico School of Medicine and Integrative Medicine Institute, Santa Fe, New Mexico, USA, ${ }^{3}$ HomInt, Karlsruhe, Germany, ${ }^{4}$ Complementary Medicine Research Unit; Primary Medical Care, University of Southhampton, Southhampton, UK, ${ }^{5} \mathrm{VSM}$ Geneesmiddelen, Alkmaar, The Netherlands, ${ }^{6} \mathrm{ClinResearch}$ GmbH, Cologne, Germany, ${ }^{7}$ Tilburg, The Netherlands, ${ }^{8}$ Institute for Complementary Medicine (KIKOM), University of Bern, Bern, Switzerland and ${ }^{9}$ passed away in 2005

Email: Max Haidvogl - max.haidvogl@meduni-graz.at; David S Riley - dsriley@integrativemed.org; Sara Brien - s.brien@soton.ac.uk; Miek Jong* - miek.jong@vsm.nl; Michael Fischer - fisher@clinr.de; George T Lewith - GL3@soton.ac.uk; Gerard Jansen - g.jansen@artsenpraktijkhomeopathietilburg.nl; André E Thurneysen - thurnand@tele2.ch

* Corresponding author †Equal contributors
\end{abstract}

This article is available from: http://www.biomedcentral.com/I472-6882/7/7

(C) 2007 Haidvogl et al; licensee BioMed Central Ltd.

This is an Open Access article distributed under the terms of the Creative Commons Attribution License (http://creativecommons.org/licenses/by/2.0), which permits unrestricted use, distribution, and reproduction in any medium, provided the original work is properly cited.

\begin{abstract}
Background: The aim of this study was to assess the effectiveness of homeopathy compared to conventional treatment in acute respiratory and ear complaints in a primary care setting.

Methods: The study was designed as an international, multi-centre, comparative cohort study of non-randomised design. Patients, presenting themselves with at least one chief complaint: acute ( $\leq 7$ days) runny nose, sore throat, ear pain, sinus pain or cough, were recruited at 57 primary care practices in Austria (8), Germany (8), the Netherlands (7), Russia (6), Spain (6), Ukraine (4), United Kingdom (10) and the USA (8) and given either homeopathic or conventional treatment. Therapy outcome was measured by using the response rate, defined as the proportion of patients experiencing 'complete recovery' or 'major improvement' in each treatment group. The primary outcome criterion was the response rate after 14 days of therapy.

Results: Data of I,577 patients were evaluated in the full analysis set of which 857 received homeopathic $(H)$ and 720 conventional (C) treatment. The majority of patients in both groups reported their outcome after 14 days of treatment as complete recovery or major improvement $(\mathrm{H}: 86.9 \%$; $\mathrm{C}: 86.0 \%$; $p=0.0003$ for non-inferiority testing). In the perprotocol set $(\mathrm{H}: 576$ and C: 540 patients) similar results were obtained $(\mathrm{H}: 87.7 \%$; $\mathrm{C}: 86.9 \%$; $p=0.0019)$. Further subgroup analysis of the full analysis set showed no differences of response rates after $\mathrm{I} 4$ days in children $(\mathrm{H}: 88.5 \%$; $\mathrm{C}$ : $84.5 \%)$ and adults $(\mathrm{H}: 85.6 \%$; $\mathrm{C}: 86.6 \%)$. The unadjusted odds ratio (OR) of the primary outcome criterion was $\mathrm{I} .40$ (0.89-2.22) in children and $0.92(0.63-1.34)$ in adults. Adjustments for demographic differences at baseline did not significantly alter the OR. The response rates after 7 and 28 days also showed no significant differences between both treatment groups. However, onset of improvement within the first 7 days after treatment was significantly faster upon homeopathic treatment both in children $(p=0.0488)$ and adults $(p=0.000 \mathrm{I})$. Adverse drug reactions occurred more frequently in adults of the conventional group than in the homeopathic group (C: $7.6 \% ; \mathrm{H}: 3.1 \%, p=0.0032)$, whereas in children the occurrence of adverse drug reactions was not significantly different $(\mathrm{H}: 2.0 \% ; \mathrm{C}: 2.4 \%, p=0.7838)$.
\end{abstract}


Conclusion: In primary care, homeopathic treatment for acute respiratory and ear complaints was not inferior to conventional treatment.

\section{Background}

The evidence base for complementary and alternative medicine (CAM) in general is limited and there is certainly a need for more research in areas such as homeopathy [1]. Objective data collection and evaluation is needed to assist physicians in patient care and advance the quality of medical practice [2]. Clinical trials, especially randomised controlled trials (RCTs), are generally accepted as producing the highest level of evidence for medical interventions. Driven by the discovery of new pharmaceutical substances, demands from regulatory authorities for clinical data and the need of physicians for evidence based treatment strategies, the methodology of RCTs became the subject of research itself. Within this context, the strengths and weaknesses of such trials have been debated [3]. Placebo-controlled RCTs are indispensable for the development of pharmaceutical agents with unknown efficacy and safety profiles. Their limitations result from highly standardized study protocols and patient populations, which may create artificial situations that differ from daily practice. Moreover, even the fact that patients are enrolled into a placebo-controlled clinical trial will influence treatment outcome, sometimes leading to high placebo or low verum response rates [4]. Consequently, more practice-based studies have been developed such as pragmatic RCT's or non-randomised cohort studies. Especially non-interventional outcomes studies have only few inclusion and exclusion criteria. Therefore they may provide information about a broad and heterogenous patient population thus resulting in high external validity for daily medical practice. However, the fact that patients are not randomly assigned to treatments in such outcome studies may lead to baseline differences between groups and makes the interpretation of the results more susceptible to bias. This disadvantage may be overcome, at least in part, by the application of statistical methods to control for baseline differences between treatment groups.

Apart from the ongoing discussion about clinical evidence, complementary therapies are well integrated into primary care in most Western countries. Among these, homeopathy is the most frequently used form in various acute and chronic conditions [5-9]. The value of homeopathy in chronic conditions has been demonstrated in several studies. A comprehensive analysis of outcome and cost-effectiveness showed that chronically ill patients had a better overall outcome with homeopathic than with conventional care [10]. Another large-scale observational study showed a positive impact of homeopathy on the health status in a substantial proportion of patients suffer- ing from a wide range of different chronic diseases [11]. To our knowledge, no large comparative cohort studies have been performed to investigate the outcome of homeopathic treatment for acute illnesses. Results of the first phase of this study, the International Integrative Primary Care Outcomes Study 1 (IIPCOS-1), suggest that homeopathic treatment is at least as effective as conventional treatment for acute complaints of the upper and lower respiratory tract [12]. The aim of the present study, IIPCOS2 , was to evaluate on an international basis and in a large sample size if homeopathic treatment is non-inferior to conventional treatment in patients with acute respiratory and ear complaints.

\section{Methods \\ Study design}

IIPCOS-2 is an international, multi-centre, comparative cohort study of non-randomised design, which was conducted between October 1998 and April 2000. Patients suffering from acute respiratory and ear complaints were recruited by physicians in 57 primary care practices in Austria (8), Germany (8), the Netherlands (7), Russia (6), Spain (6), Ukraine (4), United Kingdom (10) and USA (8). The physicians belonged to 3 different groups: providing homeopathic treatment only (22), providing either homeopathic or conventional treatment (9), and providing conventional treatment only (12). The physicians, prescribing primarily homeopathic single remedies, had in addition to their conventional medical qualifications, graduated from a homeopathic training program and at least 5 years experience using homeopathy in their medical practice. The protocol was approved by the International Ethics Committee in Freiburg, Germany. The study was conducted in accordance with the declaration of Helsinki, Good Clinical Practice (GCP) guidelines and national legal requirements.

\section{Patients}

Patients older than one month, presenting themselves with at least one of five chief complaints (runny nose, sore throat, ear pain, sinus pain or cough), and onset of symptoms not more than 7 days before, were eligible to participate. Each chief complaint comprised of 5 to 9 individual symptoms, which were rated by the physicians with scores from 0 - not present to 4 - very severe. The mean score for each chief complaint was used to measure severity at baseline. Patients meeting the inclusion criteria, respectively in case of children their parents/legal guardians, were informed by the physician about the nature of the study. Prior to enrolment into the trial each patient/parent had 
to provide written informed consent to participate. Exclusion criteria were among others severe mental impairment, severe chronic diseases such as spinal cord injuries and alcohol or drug abuse. At centres providing both therapies (mixed centres) the treatment was chosen by the physicians and/or following the patients' preference.

\section{Study protocol}

During the initial patient contact the physician documented the onset of chief complaint, severity of symptoms, clinical diagnosis, concomitant medical problems and medication and primary treatment prescribed. Patients completed a questionnaire asking for demographic and health-related information. Additionally some general questions addressed the patients' willingness to pay, patient confidence in health care provider and therapy, treatment preference, willingness to be randomized (at mixed centres only) etc. The patient follow-up was carried out by telephone 7, 14 and 28 days after the initial contact. Independent external study collaborators performed the calls. According to the study protocol they were blinded for the patient's treatment. The following parameters were documented: severity of complaintrelated symptoms, time until occurrence of first improvement, therapy outcome (assessed with complete recovery, major improvement, slight improvement, no change or deterioration), patient's satisfaction with the treatment (very satisfied, satisfied, neutral, dissatisfied or very dissatisfied) and general health condition. In case any adverse events had occurred, the physician was informed in order to collect more information and medically assess the case.

The response rates were defined as the proportion of patients assessing themselves as 'completely recovered' or 'major improved' after 7, 14 and 28 days of treatment. The main outcome criterion was the response rate after 14 days. Other outcome criteria were the response rates after 7 and 28 days, time to onset of first improvement (patients' assessments after how many days they had experienced a first improvement), patient satisfaction with treatment and health care provider and the occurrence of adverse events. Adverse events were coded by using the WHO-ART terminology.

A total of 72 selected homeopathic medications in potencies of $12 \mathrm{C}$ and higher (manufactured according to the German Homeopathic Pharmacopoeia), were given to the physicians as the basic set of study medication. Nevertheless, the physicians were free to prescribe any other remedy, any other potency or dosage form. Conventional treatment, registered in each participating country, was prescribed by the investigator and picked from a pharmacy.

\section{Data collection and monitoring}

Data were collected with a validated remote data entry system that was accessed via the Internet. The physicians entered their data online into electronic case report forms. The remote data entry system checked each entry for completeness and consistency. It recorded all data values with date and time of entry as well as all changes in the database in an audit trail. Access to the database was protected by password identification. Each user had a unique password that was provided in a sealed envelope. After entering was completed, data were transferred via Internet to the data collection centre at the former Institute for Numerical Statistics (IFNS, acquired by Omnicare Inc. in 1999) in Cologne, Germany. Monitoring was performed adherent to GCP-guidelines by an independent clinical monitor. Monitoring visits took place at least twice in order to inspect the course of the trial and to carry out source data verification. A data review tool enabled the monitor to identify missing data values, data values deviating from the normal range and among other things, data needing source verification.

\section{Statistical methods}

Data analysis was conducted by ClinResearch, Cologne, Germany, using the statistical software package SAS 9.1.3 under Windows XP Professional. The study was designed to confirm non-inferiority of the primary outcome criterion in the total patient population after homeopathic treatment in comparison to conventional treatment, using the one-sided equivalence test at the $2.5 \%$ significance level. The non-inferiority margin was defined by $5 \%$ points. Subgroup analyses were performed on age groups (children: $<18$ years; adults: $\geq 18$ years) with respect to demographic data, response rates, patient satisfaction and other outcome criteria using the Chi-square test, Fisher's exact test and Wilcoxon's rank sum test. The treatment groups were checked for baseline comparability and logistic regression analysis was performed to control for baseline differences. The primary and secondary outcome criteria were analysed on the full-set population, comprising all patients who received at least one dose of investigational medication and having at least one follow-up contact. Missing data in case of patient withdrawals from the trial were replaced by applying the last observation carried forward (LOCF) principle. A secondary analysis was performed on the per-protocol set population, comprising all patients with follow-up data on day 14 .

\section{Results \\ Patients}

A total of 2,055 patients suffering from at least one chief complaint (acute runny nose, sore throat, ear pain, sinus pain or cough) were enrolled in the study and given either homeopathic ( $\mathrm{H}: \mathrm{n}=1,220)$ or conventional treatment $(\mathrm{C}: \mathrm{n}=829)$ (Figure 1 ). Six patients did not receive any 
treatment and were excluded from further analysis. All patients from the USA and Spain $(\mathrm{H}: \mathrm{n}=216 ; \mathrm{C}: \mathrm{n}=29)$ were excluded since telephone interviews were not performed according to the study protocol. For another 227 patients no follow-up data were available because either interviews could not be carried out or the patient withdrew from the study. Data of 1577 patients with at least one follow-up contact were evaluated (full-set analysis), 857 patients in the homeopathy group and 720 patients in the conventional treatment group. For 1116 patients $(\mathrm{H}: \mathrm{n}=576 ; \mathrm{C}: \mathrm{n}=540)$ follow-up data on day 14 were documented, being the per-protocol set (Figure 1).

Upon enrolment in the study, patients, or the patients' legal guardians were asked for their treatment preference. In the homeopathy group, $81 \%$ of patients had a preference for homeopathy, $18 \%$ had no treatment preference. In the conventional group, $55 \%$ of the patients' preferred conventional treatment, $2 \%$ homeopathy and $43 \%$ had no treatment preference. Patients at mixed centres were additionally asked whether they would agree to be randomized if the choice of treatment was made randomly. With $68.1 \%$, the majority of patients in the homeopathy group refused to be randomized, $30.6 \%$ had no problem with randomisation and in $1.3 \%$ no remark was given. In the conventional group willingness and unwillingness to be randomized were equally distributed $(51.9 \%$ yes, $47.9 \%$ no, $0.1 \%$ no remark).

\section{Baseline characteristics}

Demographic data of children $(<18$ years of age $)$ and adults ( $\geq 18$ years of age) are presented in Table 1 . The proportion of children under 18 years was $47 \%$ of patients receiving homeopathic compared to 35\% receiving conventional treatment. Within this subpopulation the average age and Body Mass Index (BMI) differed significantly between both treatment groups. In adults, the distribution of males and females, average age and BMI differed significantly between the homeopathic and conventional group.

As shown in Table 2, cough was the most frequently reported chief complaint in children, followed by sore throat and ear pain. In adults sore throat was the most frequent, followed by cough and runny nose. The overall distribution of the five chief complaints in children was comparable in both treatment groups, but differed significantly in adults ( $p=0.0026$, Chi-square test). The mean severity score differed significantly at baseline for 2 out of 5 chief complaints, both in children and adults (Table 2).

With regard to the diagnosis of the chief complaints, in children otitis media was most frequently diagnosed $(\mathrm{H}$ : 18.9\%; C: $13.5 \%$ ) followed by bronchitis (H: $16.7 \%$; C: $10.7 \%$ ) and laryngitis (H: $12.3 \%$; C: $12.7 \%)$. In adults, pharyngitis (H: 23.1\%; C: 14.7\%), bronchitis (H: 11.5\%; C: $17.1 \%$ ) and tonsillitis (H: $13.9 \%$; C: $8.9 \%$ ) were most frequently diagnosed. In adults, no significant differences were observed with respect to concomitant medical problems (H: $34.2 \%$; C: $36.6 \%$ ) or concomitant medication (H: $20.7 \%$; C: $20.1 \%$ ). In the homeopathic group $21.6 \%$ of the children had concomitant medical problems versus $13.5 \%$ in conventional group ( $p=0.0098$; Fisher's exact test). The proportion of children receiving concomitant medication was higher in the homeopathic group $(9.1 \%)$ than in the conventional group $(6.7 \%)$ as well but did not reach a statistical significant level $(p=0.3098$; Fisher's exact test).

\section{Medication}

A total of 62 different homeopathic remedies were prescribed primarily on an individual basis. The top 10 (Table 3) of the most frequently prescribed homeopathic remedies included typical 'acute' remedies and accounted for about $60 \%$ of the prescriptions. In the conventional group 190 different medications were prescribed. Most of them were antibiotics followed by nasal preparations and analgesics (Table 3).

\section{Treatment outcome}

The primary outcome criterion, defined as the percentage of patients with complete recovery or major improvement after 14 days, was first calculated for the total patient population. The one-sided test of the full-set analysis showed non-inferiority of homeopathic in comparison with conventional treatment $(\mathrm{H}: 86.9 \%$; $\mathrm{C}: 86.0 \% ; p=0.0003)$. These results were confirmed by the analysis on the perprotocol set (including all patients with data at day 14) since similar response rates were obtained in both treatment groups (H: 87.7\%; C: 86.9\%; $p=0.0019$ ).

The response rates at various time points in children and adults are shown in Figure 2. The primary outcome criterion (response rate at day 14 ) in children was $88.5 \%$ after homeopathic and $84.5 \%$ after conventional treatment. In addition, response rates after 7 days (H: 68.8\%; C: 64.3\%) and 28 days (H: 93.1\%; C: 92.5\%) did not differ between both treatment groups either. In adults, the response rates after 7 days (H: 71.2\%; C: 68.8\%), 14 days (H: 85.6\%; C: 86.6\%, LOCF) and 28 days (H: 93.9\%; C: 95.9\%; LOCF) of treatment were not significantly different as well.

Since the majority of patients (> 84\%) were fully recovered or major improved after 14 days of treatment, it was of relevance to look at outcome differences within the first 7 days. As shown in Figure 3, the percentage of children experiencing a first improvement at different time points within the first week of treatment was significantly higher in the homeopathy group compared to the conventional 


\section{Homeopathy Conventional}

All patients

\section{5}

No treatment

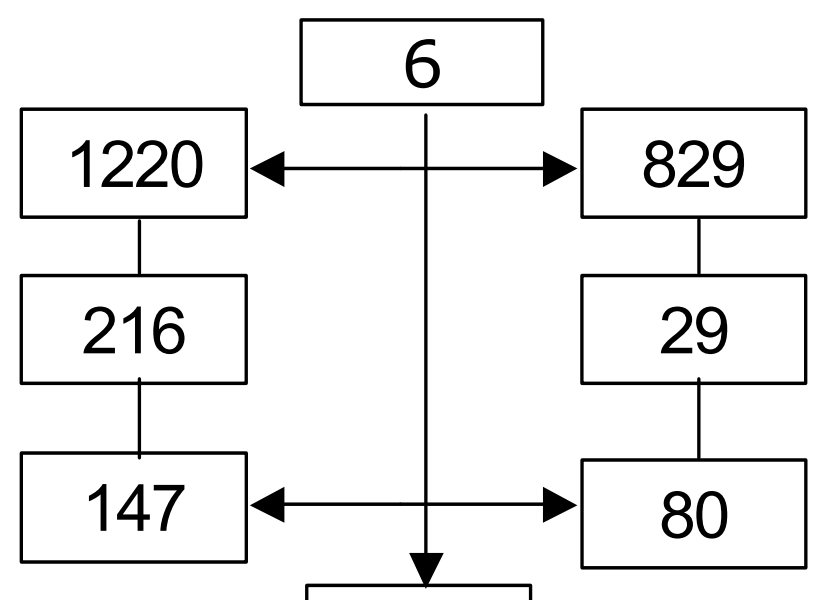

Full-Set-Analysis*

No follow-up

Excluded patients
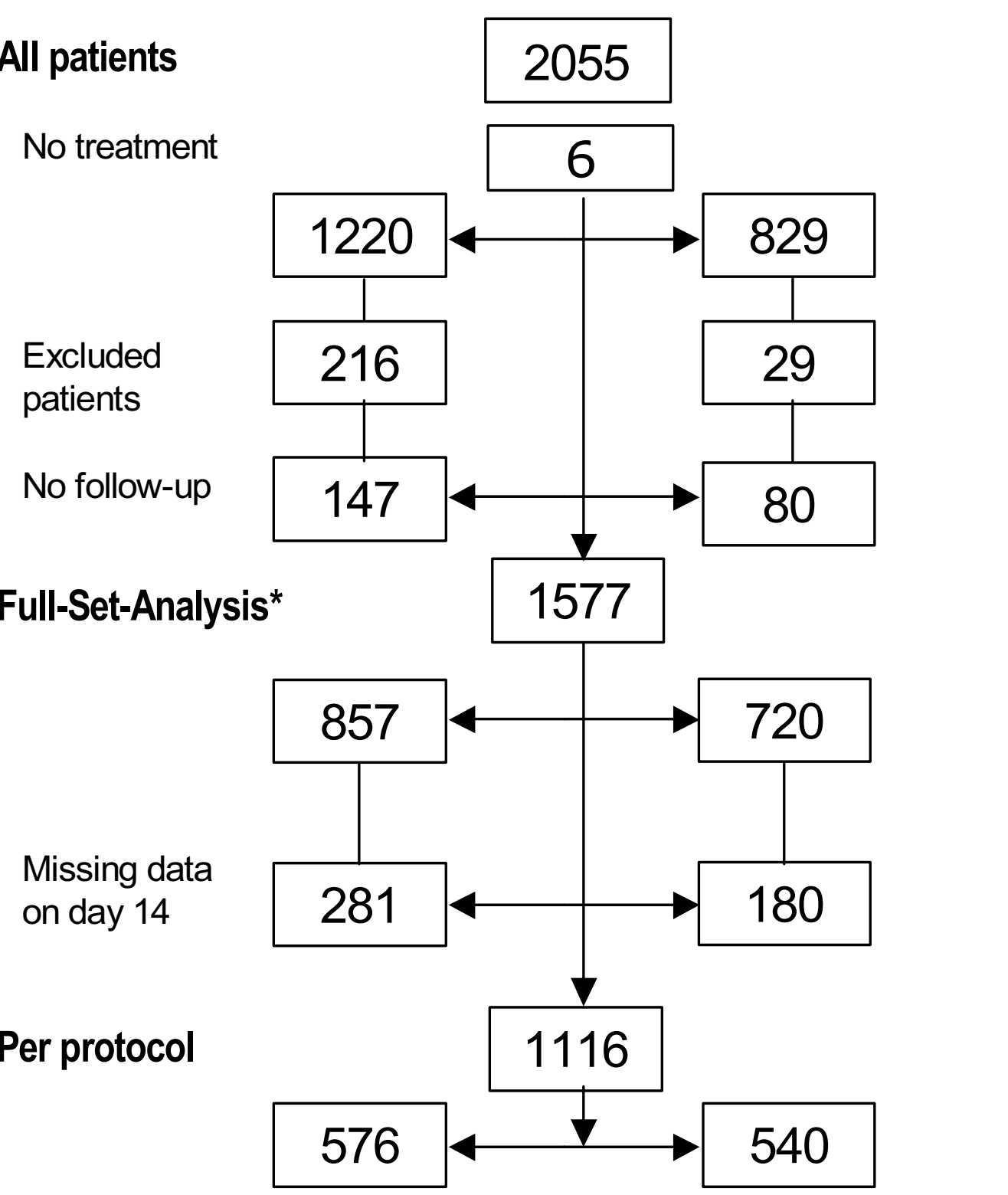

Figure I

Patient flow-chart. * All patients who received at least one dose of investigational medication and having at least one followup contact. 
Table I: Demographic data

\begin{tabular}{|c|c|c|c|}
\hline Children & Homeopathy, $n=407$ & Conventional $n=252$ & $p$-value, if $<0.05$ \\
\hline Male (\%) & 51.1 & 50.0 & \\
\hline Female (\%) & 48.9 & 50.0 & \\
\hline Age & $6.6 \pm 4.3$ & $7.4 \pm 4.7$ & $=0.0282^{\mathrm{a}}$ \\
\hline BMI & $16.6 \pm 3.0$ & $17.9 \pm 3.7$ & $=0.000 \mathrm{I}^{\mathrm{a}}$ \\
\hline Adults & Homeopathy $n=445$ & Conventional $n=462$ & $p$-value, if $<0.05$ \\
\hline Male (\%) & 24.0 & 32.3 & $=0.0064^{\mathrm{b}}$ \\
\hline Female (\%) & 76.0 & 67.7 & \\
\hline Age & $37.1 \pm 12.5$ & $39.6 \pm 13.9$ & $=0.0124^{a}$ \\
\hline BMI & $24.3 \pm 4.8$ & $25.0 \pm 4.5$ & $=0.003 \mathrm{I}^{\mathrm{a}}$ \\
\hline Smoking (\%) & 16.2 & 22.3 & \\
\hline
\end{tabular}

Full-set analysis values are either expressed as \% of total or as mean \pm SD, aWilcoxon rank-sum test, bFisher's exact test.

group ( $p=0.0488)$. For adults, a similar significant difference in favour of homeopathy $(p=0.0001)$ was observed.

Additional analysis on the primary outcome criterion in order to correct for demographic differences at baseline was carried out (Figure 4). The unadjusted odds ratio (OR) of the primary outcome criterion was $1.40(0.89-$ $2.22)$ for children and $0.92(0.63-1.34)$ for adults. In the subgroup of children, adjustments for age, mean severity and concomitant medical problems had little effect on the OR. The unadjusted OR for the Body-Mass-Index was 1.92 (1.03-3.60) and the only one showing a significant difference in favour of homeopathy. Adjustment for BMI differences between both treatment groups at baseline minimally reduced the OR to $1.89(1.00-3.57)$. In adults, individual adjustments for all variables had little to no effect on the OR of the primary outcome criterion (Figure $4)$.
Another outcome measure was the occurrence of adverse drug reactions. The percentage of children experiencing a suspected adverse drug reaction was not significantly different in both groups $(\mathrm{H}: 2.0 \%$; C: $2.4 \%, p=0.7838$, Fisher's exact test). In adults, the number of suspected adverse drug reactions was significantly higher after conventional than after homeopathic treatment (C: 7.6\%; H: $3.1 \% ; p=0.0032$, Fisher's exact test). Both in children and adults, the suspected adverse drug reactions occurred predominantly in the body as a whole (upon homeopathic treatment) or in the gastro-intestinal system (upon conventional treatment).

In addition, patients' satisfaction with treatment and healthcare provider was evaluated. Almost all patients in both treatment groups were either satisfied or very satisfied with the treatment after 28 days (children: 95\% H; $93 \%$ C, adults: 91\% H; 95\% C). A very high percentage of

Table 2: Distribution and severity score of chief complaints at Day 0

\begin{tabular}{|c|c|c|c|c|c|}
\hline \multirow{2}{*}{$\begin{array}{l}\text { Children } \\
\text { Chief complaint }\end{array}$} & \multicolumn{2}{|c|}{ Homeopathy $n=407$} & \multicolumn{2}{|c|}{ Conventional $n=252$} & \multirow[b]{2}{*}{$p$-value ${ }^{a}$ if $<0.05$} \\
\hline & $(\%)$ & Severity score & (\%) & Severity score & \\
\hline Runny nose & 9.8 & $\mathrm{I} . \mathrm{I} \pm 0.5$ & 15.5 & $1.9 \pm 0.7$ & $=0.0001$ \\
\hline Sore throat & 24.6 & $1.7 \pm 0.6$ & 23.0 & $1.6 \pm 0.6$ & \\
\hline Ear pain & 23.1 & $\mathrm{I} .4 \pm 0.6$ & 21.0 & $1.0 \pm 0.5$ & $=0.0002$ \\
\hline Sinus pain & 2.0 & $1.6 \pm 0.4$ & 3.6 & $\mathrm{I} .7 \pm 0.6$ & \\
\hline Cough & 40.5 & $0.9 \pm 0.5$ & 36.9 & $1.1 \pm 0.6$ & \\
\hline Adults & \multicolumn{2}{|c|}{ Homeopathy $n=445$} & \multicolumn{2}{|c|}{ Conventional $n=462$} & \\
\hline Chief complaint & $\%$ & Severity score & $\%$ & Severity score & $p$-value ${ }^{a}$ if $<0.05$ \\
\hline Runny nose & 15.1 & $1.5 \pm 0.8$ & 14.7 & $1.9 \pm 0.7$ & $=0.0005$ \\
\hline Sore throat & 43.4 & $1.6 \pm 0.7$ & 32.3 & $\mathrm{I} .5 \pm 0.6$ & \\
\hline Ear pain & 3.4 & $1.0 \pm 0.3$ & 5.4 & $1.3 \pm 0.5$ & \\
\hline Sinus pain & 8.3 & $1.5 \pm 0.6$ & 13.4 & $1.5 \pm 0.6$ & \\
\hline Cough & 29.9 & $1.0 \pm 0.5$ & 34.2 & $1.3 \pm 0.5$ & $=0.0002$ \\
\hline
\end{tabular}

Full-set analysis values are either expressed as \% of total or as mean \pm SD. aWilcoxon rank-sum test, indicating the differences between severity scores (from 0 - not present to 4 - very severe) in the homeopathy and conventional group. 
Table 3: The most frequently prescribed medications

\begin{tabular}{|c|c|c|c|}
\hline \multirow{2}{*}{$\begin{array}{l}\text { Children } \\
\text { Homeopathic treatment } n=407\end{array}$} & \multirow[b]{2}{*}{$\%$} & \multicolumn{2}{|l|}{ Adults } \\
\hline & & Homeopathic treatment $n=445$ & $\%$ \\
\hline I. Belladonna & 13.3 & I. Hepar sulphuris & 9.7 \\
\hline 2. Pulsatilla & 10.6 & 2. Belladonna & 8.3 \\
\hline 3. Hepar sulphuris & 6.6 & 3. Bryonia alba & 7.2 \\
\hline 4. Mercurius solubilis & 6.4 & 4. Lycopodium clavatum & 7.2 \\
\hline 5. Phosphorus & 4.9 & 5. Kalium bichromicum & 5.8 \\
\hline 6. Bryonia alba & 3.7 & 6. Mercurius solubilis & 4.9 \\
\hline 7. Calcarea carbonica & 3.7 & 7. Allium cepa & 4.5 \\
\hline 8. Lycopodium clavatum & 3.7 & 8. Phosphorus & 3.4 \\
\hline 9. Sulphur & 3.7 & 9. Causticum & 3.1 \\
\hline 10. Phytolacca decandra & 3.4 & 10. Gelsemium sempervirens & 2.7 \\
\hline Conventional treatment $n=252$ & $\%$ & Conventional treatment $n=462$ & $\%$ \\
\hline I. Antibacterials & 28.2 & I. Antibacterials & 39.4 \\
\hline 2. Nasal preparations & 22.6 & 2. Nasal preparations & 15.2 \\
\hline 3. Analgesics & 12.7 & 3. Analgesics & 9.5 \\
\hline 4. Stomatological preparations & 8.7 & 4. Cough/cold preparations & 8.7 \\
\hline 5. Anti-asthmatics & 5.6 & 5. Stomatological preparations & 5.2 \\
\hline
\end{tabular}

children (H: 98\%; C: 95\%) and adults (H: 97\%; C: 97\%) were either satisfied or very satisfied with the healthcare provider.

\section{Discussion}

The overall outcome of the first phase of the IIPCOS study [12] is confirmed in the present study on a larger group of patients and a greater number of medical practices, showing that homeopathic treatment is not inferior to conventional treatment for the treatment of acute respiratory and ear complaints. In IIPCOS- 1 the response rate of homeopathically treated patients was with $82.6 \%$ significantly higher than in the conventional group. In IIPCOS- 2 the response to homeopathic treatment was with $86.9 \%$ even higher, confirming the good effectiveness. However, no difference was observed between both treatment groups. This is due to a much higher response rate in the conventional group in IIPCOS- 2 of $86.0 \%$ compared to $68 \%$ in IIPCOS-1. One difference between both studies is that in IIPCOS-2, only patients from Europe were analysed since those recruited at practices from the USA were excluded due to protocol deviations. In IIPCOS- 1 , the majority of patients included had their residence in the USA. However, despite these differences, the overall conclusion from both studies can be drawn that homeopathy is not inferior to conventional therapy. Due to the study design, the findings of IIPCOS- 1 and IIPCOS- 2 do not provide firm data on the comparative efficacy of homeopathic and conventional treatment in acute diseases but rather underline the potential value of homeopathy in every day clinical practice. Both studies reflect the situation in every day homeopathic practice in an international setting with average patients receiving the usual treatment of a home- opathic doctor. Furthermore, patients were recruited on the basis of chief complaints and related symptoms, rather than on the clinical diagnoses. This symptomatic approach coincides with the homeopathic nature of prescription by treating each patient individually, based on specific key symptoms and patient characteristics.

In IIPCOS-2, differences for various demographic parameters and symptom-related variables were found between both groups. Thereby the profile of typical patients seeking homeopathic therapy was confirmed $[13,14]$, i.e. they were more likely to be women, younger of age, less likely to smoke and to have a lower BMI. The severity of symptoms at baseline was significantly different between treatment groups as well. However the differences were small and their clinical relevance is doubtful. Indeed regression analysis had little effect on the primary outcome criterion, showing that treatment effects were only minimally affected by selection bias. Based on the unadjusted and adjusted odds ratios of the primary outcome criterion it appears that homeopathic treatment, in comparison to conventional treatment, is more beneficial for children than adults. This observation is in accordance with previous studies in which the improvements after homeopathic treatment were greater in children than in adults $[11,13]$.

Another possible source of bias is that the outcome criteria were assessed by the patients themselves. Since it was not possible to blind patients for their treatment, potential reporting bias from patient's expectations may have influenced the outcome. On the other hand, the patients' reports were collected by independent external study col- 
Homeopathy

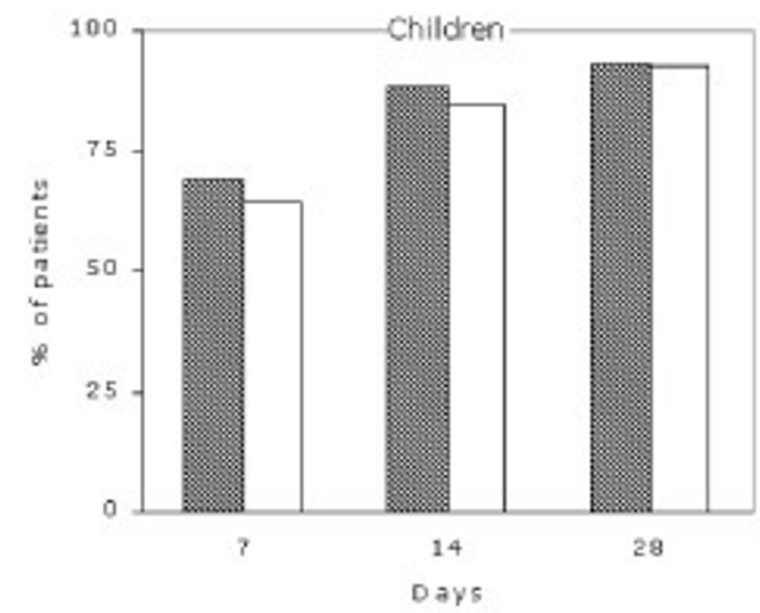

\section{Conventional}

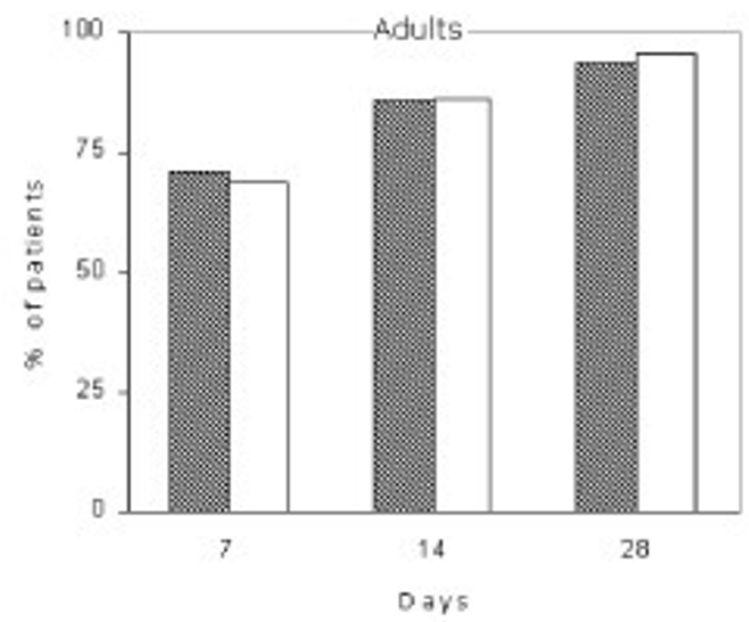

\section{Figure 2}

Response rates after 7, 14 and 28 days of treatment. Response rates (\% of patients with complete recovery or major improvement) at 7, 14 and 28 days after treatment in children and adults. Full-set analysis with last observation carried forward (LOCF) at day 14 and 28. Children $n=659$ (homeopathy, 407; conventional, 252) and adults $n=907$ (homeopathy, 445; conventional, 462).

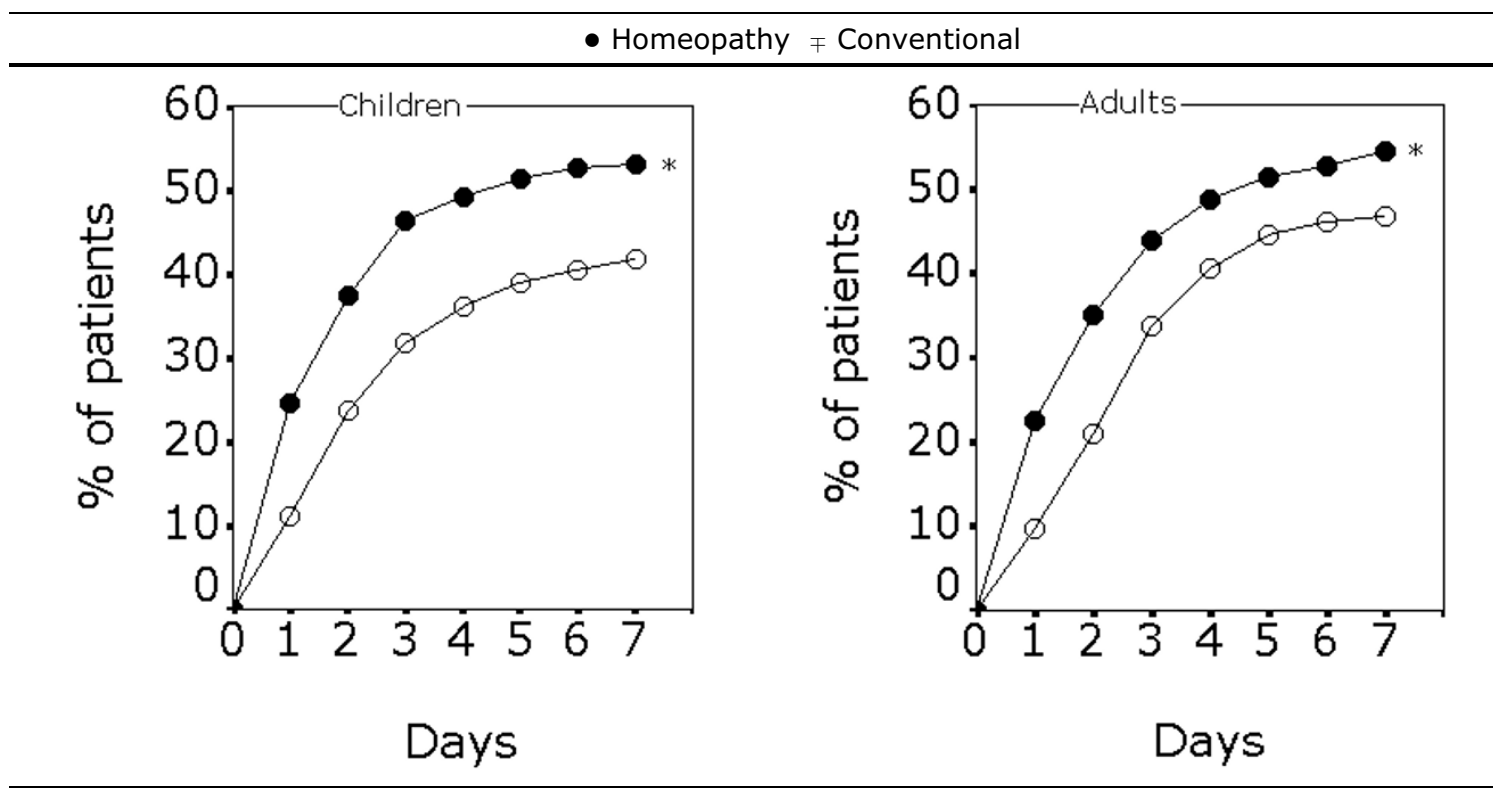

Figure 3

Onset of improvement within the first week. Onset of improvement within the first week of treatment (cumulative percentages of patients that experienced their first improvement). Children $n=659$ (homeopathy, 407; conventional, 252) and adults $n=907$ (homeopathy, 445 ; conventional, 462). Full-set analysis values with $* p=0.0448$ for children and $* p=0.000 \mathrm{I}$ for adults, using the Chi-square test on data points of the whole curve. 


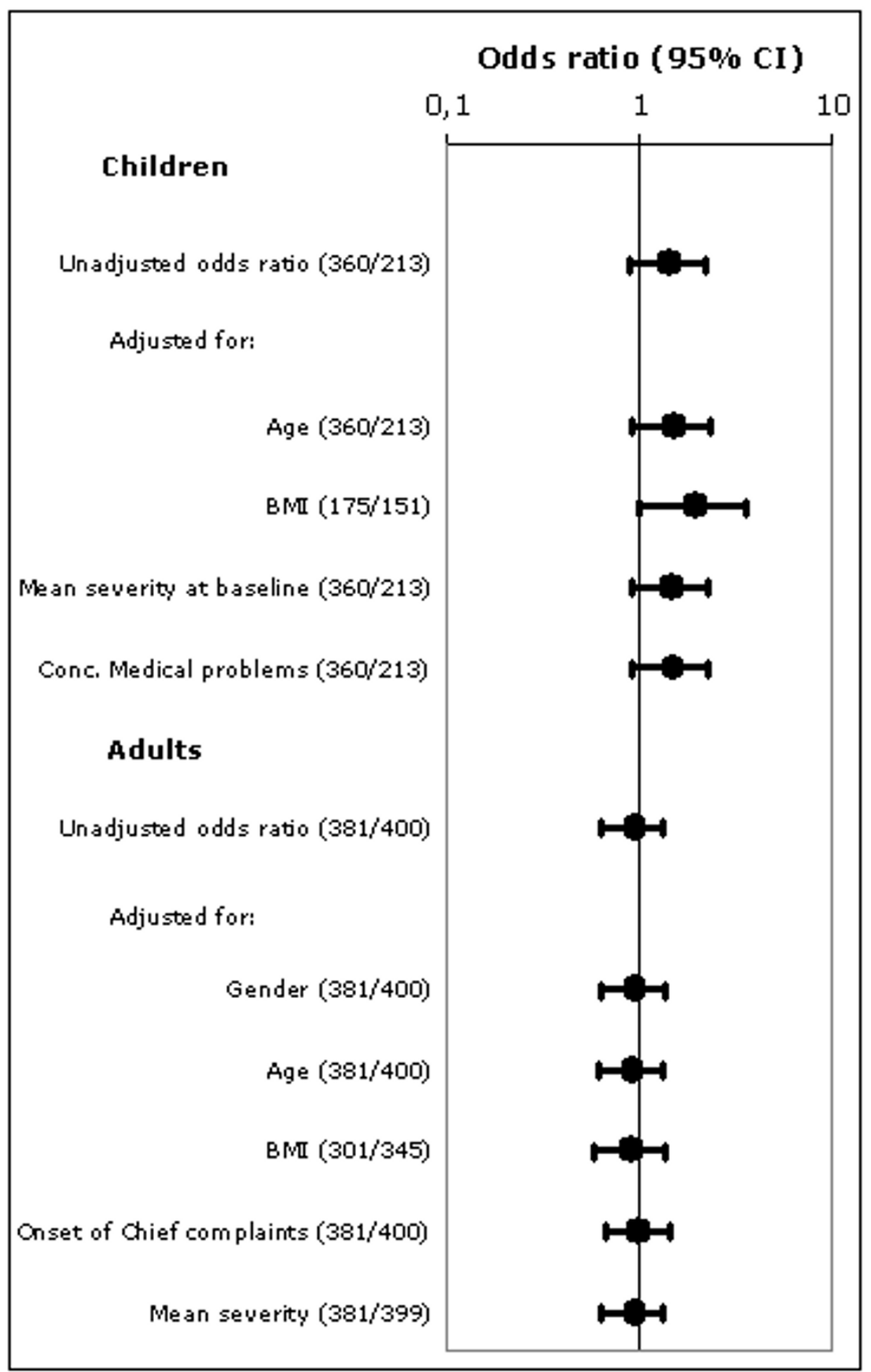

\section{Figure 4}

Main outcome measure - corrections for baseline. Main outcome measure: response to treatment (complete recovery or major improvement) of full-set analysis data at day 14, unadjusted odds ratio's and adjusted odds ratio's for baseline differences with $95 \%$ confidence intervals. Between brackets: the number of responders in the homeopathy group and conventional group, respectively. Odds ratio above I indicates a better outcome upon homeopathic treatment. 
laborators in order to minimize the influence of the patient's relationship with their physician on the treatment outcome. Although blinding of the external study coordinators was foreseen in the protocol, it cannot be ruled out that they received information from the patient revealing the nature of their medication. Therefore, blinding may not have been guaranteed in each case. Furthermore, it should be noted that at mixed centres, the choice of treatment was made by the physicians and/or following the patients' preference. The treatment decision may have been influenced by the kind or severity of the symptoms or the motivation and expectations of the patient.

Since acute respiratory and ear complaints are self-limiting conditions, it can be argued that the chosen primary outcome criterion after 14 days of treatment is not sufficiently sensitive. Patients experiencing these acute complaints may have undergone spontaneous recovery within 1 to 2 weeks. However, this outcome parameter was taken to confirm and reproduce the results of IIPCOS- 1 by using a similar study design. Therefore other outcomes criteria such as the response rate after 7 days of treatment have to be considered more carefully. Moreover, the findings that the percentage of patients experiencing a first improvement within the first week was higher at all time points in the homeopathy group than in the conventional group, are at least supportive of the 14 days finding that homeopathy is not inferior to conventional medicine.

Other observational studies on the comparability of homeopathic treatment and conventional treatment of upper respiratory tract infections (URTI) have shown positive outcomes for homeopathy $[15,16]$. Recently, the value of homeopathic treatment for the prevention of URTIs has been demonstrated in a controlled clinical trial [17]. The consistent findings in IIPCOS-1 and IIPCOS-2 further contribute to the evidence that homeopathic treatment plays a beneficial role in the primary care of patients. Furthermore, the good tolerability of homeopathic treatment of acute respiratory and ear complaints was confirmed by the low number of patients that experienced adverse drug reactions.

The major limitation of the present study is that patients were not assigned randomly to their treatment group. The majority of patients in the homeopathic group had a strong treatment preference and consequently, they were not willing to be randomized. A similar reluctance towards randomisation has also been reported elsewhere for patients seeking anthroposophic therapy [18]. These results reveal a substantial limitation to the suitability of performing large randomized controlled trials on the efficacy of homeopathy in such a primary care setting.

\section{Conclusion}

This comparative cohort study, involving more than 1,500 patients in primary care practices of at least 6 different European countries, demonstrates that homeopathic treatment for acute respiratory and ear complaints was not inferior to conventional treatment. Although no firm conclusions can be drawn about the efficacy of homeopathic treatment, these results certainly contribute to the growing evidence that homeopathy is a safe and beneficial treatment strategy for acute diseases in primary care settings.

\section{Competing interests}

$\mathrm{MJ}$ is an employee and MHe $\dagger$ was an employee of the HomInt organisation. All other authors have no financial or non-financial competing interest related to the content of the manuscript.

\section{Authors' contributions}

International Integrative Primary Care Outcomes Study 2 (IIPCOS-2) collaborators: MH, DR and MHe planned and directed the study. SB, GL, GJ and AT were responsible for data collection. MJ drafted the manuscript. MF performed the statistical analysis. All authors read and approved the final version of the manuscript.

\section{Acknowledgements}

This study was carried out by the Homlnt organisation, Karlsruhe, Germany. The IIPCOS-2 collaborators would like to thank Sytze de Roock for preparation of tables and figures, Rolf Hövelmann for data analysis, Wolfgang Mayer for monitoring the study and Rainer Lüdtke for his comments on the drafted manuscript. We are especially grateful to the physicians and all the patients for participating in the study.

\section{References}

I. Shang A, Huwiler-Müntener K, Nartey L, Jüni P, Dörig S, Sterne JA, Pewsner $D$, Egger $M$ : Are the clinical effects of homoeopathy placebo effects? Comparative study of placebo-controlled trials of homoeopathy and allopathy. The Lancet 2005, 366:726-732.

2. Bell IR: Evidence-based homeopathy: Empirical questions and methodological considerations for homeopathic clinical research. AJHM 2003, 96: I7-3I.

3. Walach H, Falkenberg T, Fonnebø V, Lewith G, Jonas WB: Circular Instead of Hierarchical: Methodological Principles for the Evaluation of Complex Interventions. BMC Med Res Methodol 2006, 6:29.

4. Walach $\mathrm{H}$, Jonas WB, Lewith $\mathrm{G}$ : The role of outcomes research in evaluating complementary and alternative medicine. Altern Ther Health Med 2002, 8:88-95.

5. Baron SE, Goodwin RG, Nicolau N, Blackford S, Goulden V: Use of complementary medicine among outpatients with dermatologic conditions within Yorkshire and South Wales, United Kingdom. J Am Acad Dermatol 2005, 52:589-594.

6. Passalacqua G, Compalati E, Schiappoli M, Senna G: Complementary and alternative medicine for the treatment and diagnosis of asthma and allergic diseases. Monaldi Arch Chest Dis 2005, 63:47-54.

7. Breuer GS, Orbach $H$, Elkayam O: Perceived efficacy among patients of various methods of complementary alternative medicine for rheumatologic diseases. Clin Exp Rheumatol 2005, 23:693-696.

8. Bensoussan M, Jovenin N, Garcia B, Vandromme L, Jolly D, Bouche O, Thiefin G, Cadiot G: Complementary and alternative medicine 
use by patients with inflammatory bowel disease: results from a postal survey. Gastroenterol Clin Biol 2006, 30: I4-23.

9. Weber U, Lüdtke R, Friese KH, Fischer I, Moeller H: A Non-Randomised Pilot Study to Compare Complementary and Conventional Treatment of Acute Sinusitis. Forsch Komplementärmed Klass Naturheilkd 2002, 9:99-104.

10. Witt C, Keil T, Selim D, Roll S, Vance W, Wegscheider K, Willich SN: Outcome and costs of homeopathic and conventional treatment strategies: A comparative cohort study in patients with chronic disorders. Complement Ther Med 2005, 13:79-86.

II. Spence DS, Thompson EA, Barron SJ: Homeopathic treatment for chronic disease: A 6-year, university-hospital outpatient observational study. J Altern Complement Med 2005, I I:793-798.

12. Riley D, Fischer M, Singh B, HaidvogI M, Heger M: Homeopathy and conventional medicine: an outcomes study comparing effectiveness in a primary care setting. J Altern Complement Med 200I, 7:149-159.

13. Witt CM, Lüdtke R, Baur R, Willich SN: Homeopathic medical practice: Long-term results of a cohort study with $398 \mathrm{I}$ patients. BMC Public Health 2005, 5: II5.

14. Kaiser S: Comparison, using psychological constructs, between patients treated with orthodox medicine and patients treated with homeopathy. Homlnt R\&D Newsletter 1998, I:I4-18.

15. Trichard M, Chaufferin G, Nicoloyannis N: Pharmacoeconomic comparison between homeopathic and antibiotic treatment strategies in recurrent rhinopharyngitis in children. Homeopathy 2005, 94:3-9.

16. Friese $\mathrm{KH}$, Kruse F, Lüdtke R, Möller H: The homeopathic treatment of otitis media in children - comparisons with conventional therapy. Int I Clin Pharmacol Ther 1997, 35:296-30I.

17. Steinsbekk A, Fonnebo V, Lewith G, Bentzen N: Homeopathic care for the prevention of upper respiratory tract infections in children: A pragmatic, randomised, controlled trial comparing individualised homeopathic care and waiting-list controls. Complement Ther Med 2005, 13:23।-238.

18. Hamre HJ, Fischer M, Heger M, Riley D, Haidvogl M, Baars E, Bristol E, Evans M, Schwarz R, Kiene H: Anthroposophic vs. conventional therapy of acute respiratory and ear infections: a prospective outcomes study. Wien Klin Wochenschr 2005, I 17:256-268.

\section{Pre-publication history}

The pre-publication history for this paper can be accessed here:

http://www.biomedcentral.com/1472-6882/7/7/prepub

\section{Publish with Bio Med Central and every scientist can read your work free of charge}

"BioMed Central will be the most significant development for disseminating the results of biomedical research in our lifetime. "

Sir Paul Nurse, Cancer Research UK

Your research papers will be:

- available free of charge to the entire biomedical community

- peer reviewed and published immediately upon acceptance

- cited in PubMed and archived on PubMed Central

- yours - you keep the copyright 